\title{
Avian Eggshell Slurry as a Dentin Desensitizing Agent: An In Vitro Assessment Using Two Techniques
}

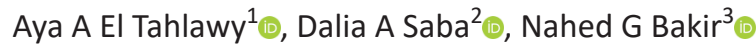

\begin{abstract}
Aim: This study aims to evaluate the efficacy of avian eggshell slurry as a desensitizing agent compared to casein phosphopeptide-amorphous calcium phosphate (CPP-ACP) containing Tooth Mousse.

Materials and methods: Eggshell powder was prepared and characterized using a scanning electron microscope (SEM), transmission electron microscope (TEM), X-ray diffraction (XRD), and energy-dispersive X-ray spectroscopy (EDX). Forty dentin disks were immersed in $6 \%$ citric acid for 2 min to simulate hypersensitive dentin (baseline). Disks were then divided into two groups $(n=20)$ according to treatment received: eggshell slurry and commercially available Tooth Mousse desensitizing agent. Each group was further divided into two subgroups ( $n=10)$. In subgroup 1, dentin discs were immersed in artificial saliva for four weeks whereas, in subgroup 2, discs underwent acid challenge. Environmental scanning electron microscope (ESEM) analysis and hydraulic conductance test were utilized to calculate dentinal tubules occlusion and reduction of dentin permeability percentages, respectively. Statistical analysis was performed using SPSS 22.0 at a significance level $p \leq 0.05$.

Results: After application of desensitizing agents, the eggshell slurry group showed a significantly higher percentage of dentinal tubules occlusion compared to the Tooth Mousse group, whereas no significant difference existed in the percentage reduction of dentin permeability between both groups. After both immersion protocols, the eggshell slurry significantly occluded more dentinal tubules compared to Tooth Mousse. On the contrary, results of percentage reduction of dentin permeability revealed no significant difference between both subgroups after acid challenge. After artificial saliva immersion, Tooth Mousse showed a significantly higher percentage reduction of dentin permeability compared to the eggshell slurry.
\end{abstract}

Conclusion: Avian eggshell slurry can effectively occlude open dentinal tubules compared to commercially available Tooth Mousse desensitizing agent.

Clinical significance: Avian eggshell slurry can be considered a promising material for the treatment of dentin hypersensitivity.

Keywords: Dentin hypersensitivity, Dentin permeability, Dentinal tubules occlusion, Eggshell, In vitro study.

The Journal of Contemporary Dental Practice (2021): 10.5005/jp-journals-10024-3086

\section{INTRODUCTION}

Dentin hypersensitivity $(\mathrm{DH})$ is a common clinical problem characterized by sharp pain lasting for a shorter period. It may result from chemical, mechanical, or thermal stimuli, which would normally cause no response in a healthy tooth. ${ }^{1}$ It could be best explained by Brännström's hydrodynamic theory, which states that movement of fluid inside dentinal tubules will stimulate the nerve endings causing pain. ${ }^{2}$ Topical application of desensitizing agents have been approached frequently to decrease the dentinal fluid movement and thereby decrease $\mathrm{DH} .{ }^{3}$ Although there is a wide range of desensitizing agents, yet there is no gold standard that permanently occludes dentinal tubules. ${ }^{4}$

Recently, there is a tremendous shift towards the usage of natural products in the dental field. Eggshell is a waste product that annually weighs tons and favors pollution. ${ }^{5}$ Eggshell has been used as a biodegradable bone substitute for the repair of bone defects. ${ }^{6,7}$ It has also been used for remineralization of early enamel carious lesions. ${ }^{8}$

Recent studies investigated the incorporation of eggshell in calcium hydroxide cement to increase its bioactivity, ${ }^{9}$ glass ionomer to enhance its mechanical properties, ${ }^{10}$ reducing the surface roughness of dental acrylic resins, ${ }^{11}$ and nano-textured scaffolds. ${ }^{12}$ Its use as a pulp capping material has also been studied. ${ }^{13}$

Owing to the great similarity between dentin and bone, it could be possible to use eggshell for dentinal tubules occlusion. Therefore, this study aimed to prepare and characterize eggshell
${ }^{1-3}$ Biomaterials Department, Faculty of Dentistry, Cairo University, Cairo, Egypt

Corresponding Author: Aya A El Tahlawy, Biomaterials Department, Faculty of Dentistry, Cairo University, Cairo, Egypt, Phone: +20 1001071865, e-mail: ayaadel@dentistry.cu.edu.eg

How to cite this article: El Tahlawy AA, Saba DA, Bakir NG. Avian Eggshell Slurry as a Dentin Desensitizing Agent: An In Vitro Assessment Using Two Techniques. J Contemp Dent Pract 2021;22(5):532-537.

Source of support: Nil

Conflict of interest: None

powder using a scanning electron microscope (SEM), transmission electron microscope (TEM), X-ray diffraction (XRD), and energydispersive X-ray spectroscopy (EDX), and to evaluate the efficacy of eggshell slurry in occluding dentinal tubules in comparison to the commercially available CPP-ACP desensitizing agent (Tooth Mousse) for treatment of dentin hypersensitivity. The null hypothesis was that there is neither difference in the percentage of dentinal tubules occlusion nor the percentage reduction in dentin permeability, between the prepared eggshell slurry and Tooth Mousse.

\section{Materials and Methods}

This study was conducted in the Biomaterials Department, Faculty of Dentistry, Cairo University, Egypt. 


\section{Eggshell Powder Preparation}

Twenty white avian chicken (Gallus gallus domesticus) eggs free of any discoloration or cracks were purchased from the local market. The Eggs were broken manually, and the albumen and yolk were kept aside to be used for other purposes. The outer and inner shell membranes of the eggshells were carefully removed using a dental tweezer. ${ }^{6}$ The eggshells were rinsed with distilled water and dried overnight at room temperature $\left(24 \pm 1^{\circ} \mathrm{C}\right) .^{14}$ The eggshells were then dry-milled in a zirconia ball milling machine (Planetary Ball Mill, Pulverisette 6, FRITSCH, Germany) at 200 rpm for 6 h. ${ }^{15}$ Afterwards, the produced powder (weighed $110 \mathrm{~g}$ ) was autoclaved at $136^{\circ} \mathrm{C}$ for 18 min for sterilization. ${ }^{16}$

\section{Characterization of Eggshell Powder}

The morphological characteristics of $0.1 \mathrm{~g}$ of eggshell powder were examined using high-resolution scanning electron microscopy (SEM) [Model Quanta 250 FEG (Field Emission Gun), FEl Company, Netherlands] coupled with energy-dispersive X-ray spectroscopy (EDX) for assessment of the elemental composition. Powder particle size was determined using a high-resolution transmission electron microscope (TEM) (JEOL JEM-1010, USA) and operating at an acceleration voltage of $70 \mathrm{kV}$. About $0.1 \mathrm{~g}$ of eggshell powder was dispersed in ethanol and a drop of the dispersion was placed on carbon-coated copper grids (CCG). The sample was then dried and analyzed at a magnification of 120000x. An X-ray diffractometer (XRD) (PANalytical X-ray diffraction equipment model X'Pert PRO) was used to identify the crystal structure of $2 \mathrm{~g}$ of the prepared powder. XRD with Cu radiation $(\lambda=1.542 \AA$ ) was used at $45 \mathrm{kV}, 35 \mathrm{~mA}$, and a scanning speed of $0.040 / \mathrm{s}$. The diffraction peaks between $2 \theta=2^{\circ}$ and $60^{\circ} .{ }^{17}$

\section{Sample Size Calculation}

Based on a previous paper by Thanatvarakorn et al. ${ }^{18}$ using the dentin permeability test, the results of occlusion of dentinal tubules within each group were normally distributed with a standard deviation of 5.4. If the true difference in the experimental and control means is 8 , then 36 teeth ( 9 in each subgroup) will be required in this study to be able to reject the null hypothesis with probability (power) 0.85 . The type I error probability associated with this test of the null hypothesis is 0.05 . Sample size calculation was carried out using the PS program. The same specimens were used for the evaluation of both dentinal tubules occlusion and reduction of dentin permeability percentages analyses. ${ }^{18}$

\section{Specimen Preparation}

A total of 40 intact freshly extracted human third molars, free from coronal caries and any developmental anomalies, were selected. The teeth were surgically extracted due to impaction and were used after the approval of the ethical committee (no. 17-2-2). Mid coronal dentin discs; $1.0 \pm 0.1 \mathrm{~mm}$ thick, were cut perpendicular to the long axis of the tooth away from the pulp chamber. ${ }^{2,19,20}$ Dentin discs were then polished, and the occlusal surface of each disc was partially covered by nail varnish leaving an exposed $5 \mathrm{~mm}$ diameter round window for standardization of the area to be measured. ${ }^{20}$ To simulate hypersensitive dentin with open dentinal tubules, the dentin discs were etched using $6 \%$ citric acid $(\mathrm{pH}=2)$ for 2 min. Ultrasonication of the discs for 5 min then followed. ${ }^{20}$

\section{Application of the Desensitizing Agents}

The dentin discs stored in sequentially numbered vials were randomly assigned by the principal investigator into two groups (20 discs/ group) using the random.org site (https://www.random.org/). The two groups were the Tooth Mousse desensitizing agent (GC dental products corp., Japan) group and the eggshell group; where the dentin discs were treated with eggshell slurry; prepared in a ratio of $1 \mathrm{mg}$ eggshell powder to $5 \mu \mathrm{L}$ distilled water.

Each agent was applied once and rubbed on the dentin disc for 1 min using a microbrush. The treated specimens were left untouched for $30 \mathrm{~min}$ and were then rinsed with distilled water. This was done to simulate the clinical situation where the patient must avoid ingestion of any kind of food or drinks for at least $30 \mathrm{~min}$ after application of the desensitizing agent. ${ }^{1}$

The specimens in each group were further randomly divided into two subgroups ( $n=10$ ) according to the immersion protocol. In the first subgroup, the artificial saliva immersion; the treated specimens were immersed in artificial saliva, prepared following the method by Lata et al. $^{21}$ for four weeks at $37^{\circ} \mathrm{C}$ to evaluate the long-term efficacy and durability of the desensitizing agents. ${ }^{22,23}$ During the immersion period, the artificial saliva was replenished every week. ${ }^{18}$ In the second subgroup, the acid challenge; the treated specimens were immersed once in $6 \%$ citric acid $(\mathrm{pH}=2)$ for $1 \mathrm{~min}$ followed by rinsing with distilled water for $2 \mathrm{~min} .^{2,19} \mathrm{Citric}$ acid was chosen as it is a common ingredient in soft drinks, fruit juices, and fruits. Therefore, $6 \mathrm{wt} \%$ citric acid $(\mathrm{pH}=2)$ was used to simulate the acidic diet and to investigate the resistance of both desensitizing agents to dietary acid conditions. ${ }^{19}$

\section{Measurement of Dentinal Tubule Occlusion Using Environmental Scanning Electron Microscopy (ESEM)}

ESEM analysis was carried out by blinded assessor after each of the following stages: simulation of hypersensitive dentin, application of the desensitizing agent, and immersion in either artificial saliva for 4 weeks or acid challenge.

Images were captured at a constant magnification of 4000x. Image analysis was performed quantitively using an image analyzer (ImageJ, NIH, USA) to measure the total surface area of the open dentinal tubules within the ESEM image of each specimen surface. The total area of open dentinal tubules following simulation of hypersensitive dentin was considered the baseline value. This value was used to calculate the percentage of occlusion, i.e., the percentage of reduction in area of dentinal tubules after the application of the agents and following the different immersion protocols.

\section{Measurement of Dentin Permeability}

Dentin permeability was measured by a blinded assessor; using the same dentin disc specimens utilized for ESEM examination, at each of the previously mentioned stages. Dentin permeability was measured by the filtration of deionized water and expressed in terms of hydraulic conductance using a hydraulic conductance system. The hydraulic conductance system was custom-made at the Faculty of Engineering similar to the Pashley and Galloway design. ${ }^{24}$ Dentin permeability was evaluated at a simulated pulpal pressure of 10 psi. $^{2,25-27}$

Each dentin disc was positioned between two rubber " $\mathrm{O}$ " rings and a split chamber uncovering the exposed $5 \mathrm{~mm}$ diameter window surrounded by the nail varnish, leaving $0.196 \mathrm{~cm}^{2}$ of the available area for filtration of the deionized water. The movement of the air bubble that is trapped inside the $3 \mathrm{~mm}$ diameter glass pipette tube; horizontally connected to the split-chamber, was recorded every minute for three consecutive minutes. The volumetric displacement of the air bubble was calculated in order to calculate the filtration rate and hydraulic conductance $(L p)$ following the equation $L p=Q / P(S A)$, where $L p$ is the hydraulic conductance of dentin in

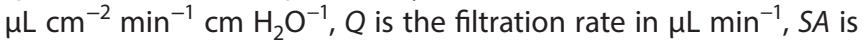


the dentin surface area in $\mathrm{cm}^{2}\left(0.196 \mathrm{~cm}^{2}\right)$, and $P$ is the hydrostatic pressure difference across dentin in $\mathrm{cm} \mathrm{H}_{2} \mathrm{O}\left(=703.1 \mathrm{~cm} \mathrm{H}_{2} \mathrm{O}\right) .{ }^{18,27,28}$

The initial hydraulic conductance value after simulation of hypersensitive dentin was considered the baseline value ( $L p$ baseline) representing the maximum permeability. After application of either agent and following each immersion protocol, the permeability reduction percentage (PR\%) was calculated for each specimen using its $L p$ baseline. ${ }^{2,18,26}$

\section{Statistical Analysis}

Statistical analysis was performed using IBM SPSS Statistics for Windows, Version 22.0. Armonk, NY: IBM, Corp. To compare the two desensitizing agents, repeated measures ANOVA test was used for parametric data whereas Mann-Whitney $U$ test was used for non-parametric data. Spearman's correlation coefficient was used to determine the correlation between the results of percentage of dentinal tubules occlusion using ESEM and those of percentage reduction of dentin permeability using hydraulic conductance system. The significance level was set at $p \leq 0.05$.

\section{Results}

\section{Characterization of the Powder}

SEM micrographs of the prepared eggshell powder showed that the powder is formed of rough and irregular particles

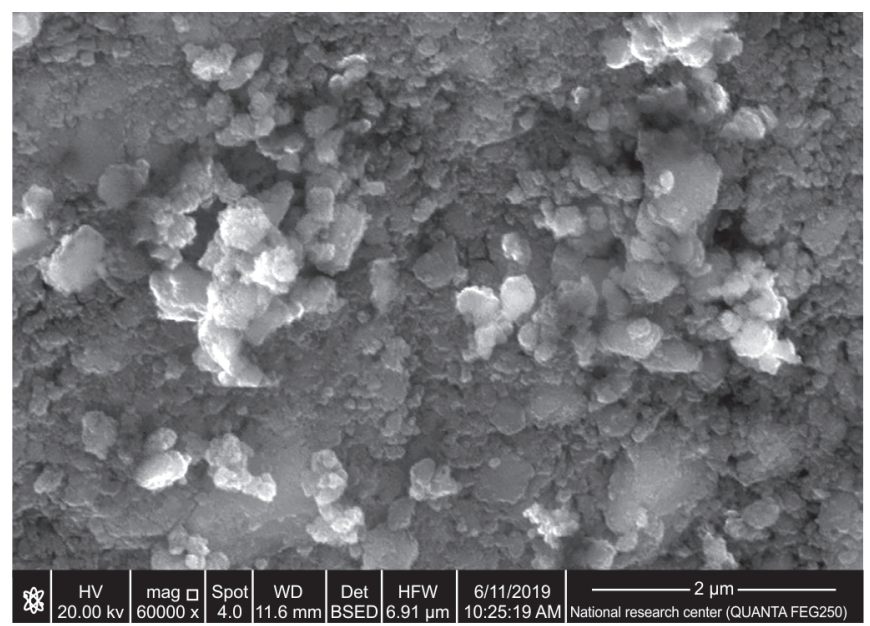

Fig. 1: SEM of the prepared eggshell powder at $60000 \times$ magnification
(Fig. 1). EDX elemental analysis revealed the presence of calcium (51.09 wt\%), oxygen (40.07 wt\%), and carbon (8.84 wt\%). The XRD pattern (Fig. 2) shows all diffraction peaks characteristic of calcite $\left(\mathrm{CaCO}_{3}\right)$. TEM micrographs (Fig. 3) revealed that the irregular eggshell powder particles observed in the SEM micrographs are agglomerates of irregularly shaped nano-sized particles. The mean particle size of ten particles measured at a magnification of $120000 \times$ was equal to $22.86 \mathrm{~nm}$ with a maximum size of $32.53 \mathrm{~nm}$ and a minimum size of $16.22 \mathrm{~nm}$.

\section{Percentage of Dentinal Tubules Occlusion}

The SEM micrographs for eggshell slurry and Tooth Mousse groups at different stages are shown in Figures 4 and 5, respectively. Repeated measures ANOVA test revealed that the prepared eggshell slurry group showed significantly ( $p$-value $<0.001$ ) higher mean percentage of dentinal tubules occlusion after application of the agents and after both immersion protocols (artificial saliva immersion and acid challenge) compared to that of the Tooth Mousse group (Table 1).

\section{Percentage Reduction of Dentin Permeability}

Mann-Whitney $U$ test revealed that no statistically significant difference in the median values of percentage reduction of dentin permeability existed between both groups after application of the agents and acid challenge. However, it revealed that the Tooth Mousse showed a significantly ( $p$-value $<0.030$ ) higher median percentage reduction of dentin permeability than that of the prepared eggshell slurry after immersion in artificial saliva (Table 2).

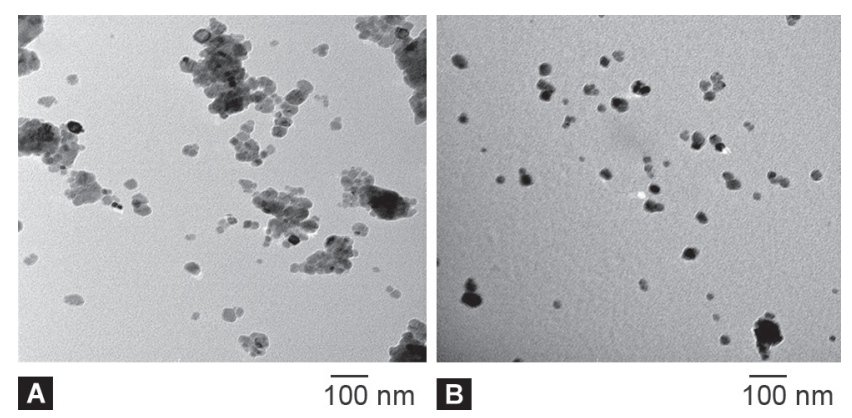

Figs 3 A and B: TEM of the prepared eggshell powder at $120000 \times$ magnification showing: (A) Agglomerates of nanoparticles; (B) Dispersed nanoparticles

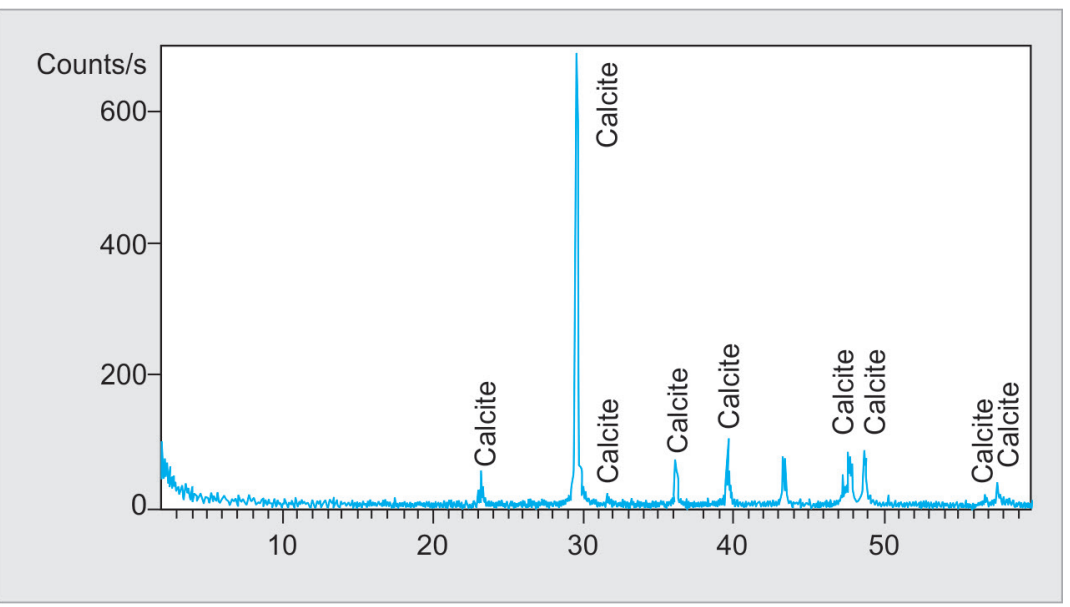

Fig. 2: XRD pattern of the prepared eggshell powder 

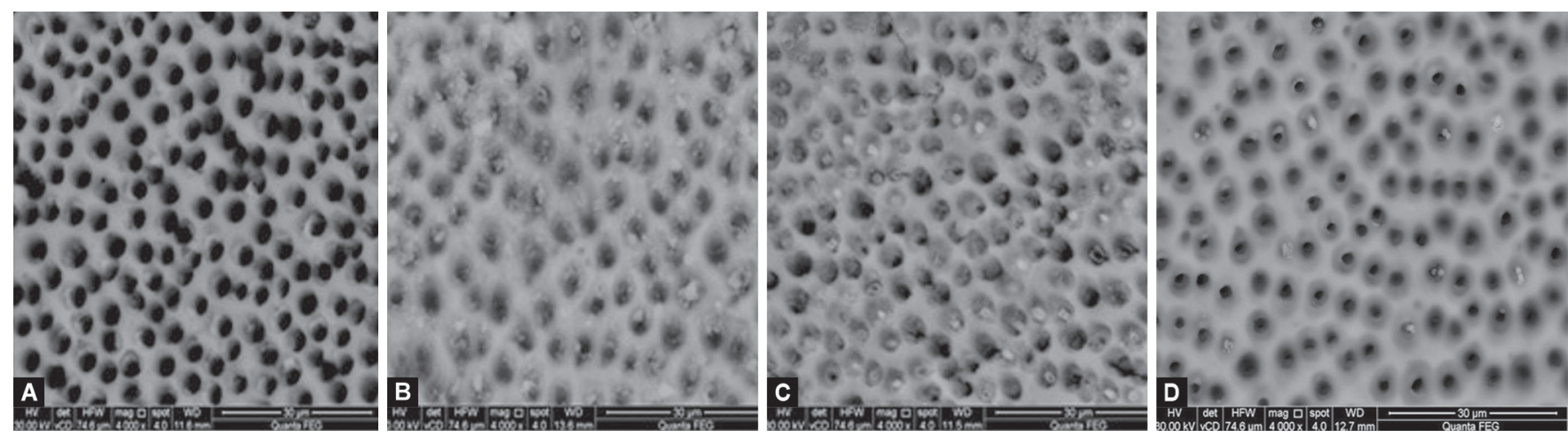

Figs 4A to D: ESEM micrograph of a dentin disc in the eggshell slurry group at a 4000× magnification: (A) Before application of the agent; (B) After application of the agent, (C) After artificial saliva immersion; (D) After acid challenge
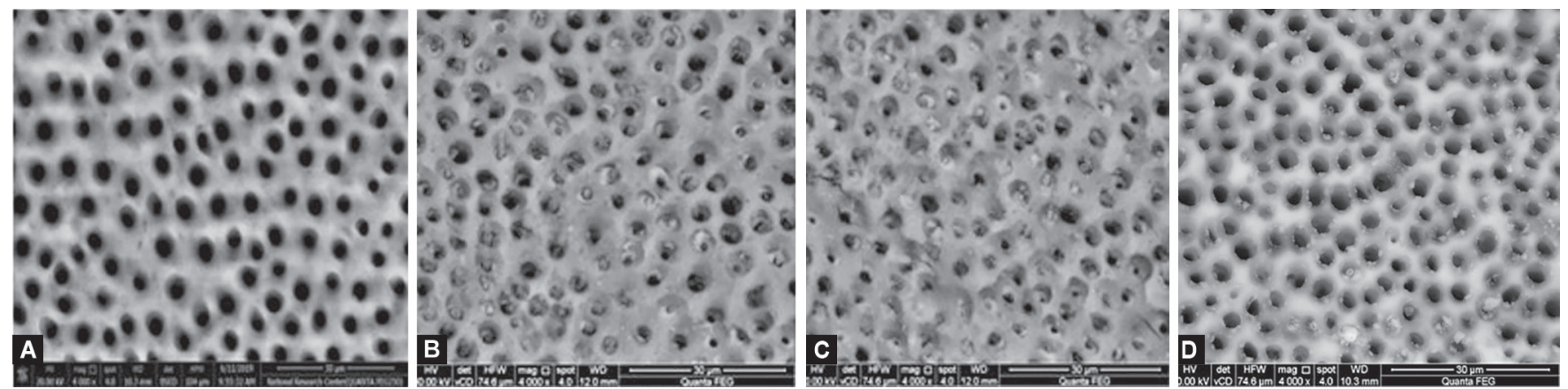

Figs 5A to D: ESEM micrograph of a dentin disc in the Tooth Mousse group at a 4000× magnification: (A) Before application of the agent; (B) After application of the agent; (C) After artificial saliva immersion; (D) After acid challenge

Table 1: Means, standard deviation (SD) values, and the results of repeated measures ANOVA test for percentage of dentinal tubules occlusion values

\begin{tabular}{|c|c|c|c|}
\hline & Prepared eggshell slurry & Tooth Mousse & \multirow[b]{2}{*}{$p$-value } \\
\hline & Mean $\pm S D$ & Mean $\pm S D$ & \\
\hline $\begin{array}{l}\text { After application } \\
\text { of the agent }\end{array}$ & $77.4( \pm 2.5)^{\dagger}$ & $61.8( \pm 3.8)^{\dagger}$ & $<0.001$ \\
\hline $\begin{array}{l}\text { Immersion in } \\
\text { artificial saliva }\end{array}$ & $77.6( \pm 2.8)^{\ddagger}$ & $65.0( \pm 3.1)^{\ddagger}$ & $<0.001$ \\
\hline Acid challenge & $65.0( \pm 4.2)^{\ddagger}$ & $56.0( \pm 4.5)^{\ddagger}$ & $<0.001$ \\
\hline
\end{tabular}

*Statistically significant at $p \leq 0.05$; ${ }^{\dagger}$ Mean \pm SD of 20 dentin discs; ${ }^{\ddagger}$ Mean \pm SD of 10 dentin discs

Table 2: Median, range values, and the results of the Mann-Whitney $\mathrm{U}$ test for percentage reduction of dentin permeability

\begin{tabular}{llllll}
\hline \multicolumn{5}{c}{$\begin{array}{l}\text { Prepared } \\
\text { eggshell slurry }\end{array}$} & \multicolumn{1}{l}{ Tooth Mousse } \\
\cline { 2 - 6 } & Median & Range & Median & Range & $p$-value \\
\hline $\begin{array}{l}\text { After application } \\
\text { of the agent }\end{array}$ & $50^{* *}$ & $33.3-100$ & $50^{* *}$ & $25-100$ & 0.475 \\
$\begin{array}{l}\text { After immersion } \\
\text { in artificial saliva }\end{array}$ & $50^{+\dagger}$ & $40-66.7$ & $75^{\text {t+ }}$ & $50-100$ & $0.030 \S$ \\
$\begin{array}{l}\text { After acid chal- } \\
\text { lenge }\end{array}$ & $33.3^{+\dagger}$ & $0-50$ & $33.3^{+\dagger}$ & $0-50$ & 0.590
\end{tabular}

${ }^{*}$ Statistically significant at $p \leq 0.05 ;{ }^{* *}$ Median of 20 dentin discs; ${ }^{+\dagger}$ Median of 10 dentin discs

When the overall results of percentage of dentinal tubules occlusion using ESEM after the different stages were correlated with those of the dentin permeability using the hydraulic conductance system, insignificant negative correlation existed between them $(\rho=-0.2185, p$-value $=0.514)$.

\section{Discussion}

Dentin hypersensitivity is a common and prevalent dental disease confronted frequently in clinical practice. As opposed to periodontal diseases or dental caries, dentin hypersensitivity is one of the most painful and not easily treated dental problems affecting the quality of patient's life. ${ }^{29}$

Since it has been proven that eggshell is a promising material for the repair of bone defects and due to similarity between bone and dentin, the use of eggshell as dentin desensitizing agent was thought of. The usage of eggshell waste may aid in reducing the usage of other expensive calcium sources, i.e., cost-effective. ${ }^{30,31}$ From an environmental sustainability and management perspective, using eggshell waste material to treat dentin hypersensitivity may serve as a pathway for recycling this material. This in turn may strengthen the economic benefits associated with using a natural waste material, which is high on the global agenda for a greener environment. $^{32}$

The results of the present study revealed that a significant difference in dentinal tubule occlusion and percentage reduction in dentin permeability existed between some of the tested eggshell slurry and Tooth Mousse subgroups. Therefore, the null hypothesis was rejected.

The ESEM analysis conducted in the present study to measure the dentinal tubule occlusion has the advantage of detecting the changes in dentin surface morphology of the same sample throughout the different experimental stages without any sample preparation. The image analysis program (Image J); used to measure 
the total area of open dentinal tubules, was another advantage. Image J program reduces the subjectivity of the analysis and allows quantitative rather than qualitative analysis. ${ }^{20,33}$

Following the application of the eggshell slurry, a significant increase in the mean percentage of dentinal tubules occlusion was revealed. This may be related to both the chemical composition and the particle size of the prepared eggshell powder. Characterization of the prepared eggshell powder via XRD analysis revealed the presence of all diffraction peaks characteristic of calcite, $\mathrm{CaCO}_{3}$, which is in agreement with the findings of Bashir and Manusamy, and those of Freire et al. ${ }^{17,34}$ This was further confirmed by the EDX elemental analysis where all the EDX peaks were identified as calcium, oxygen, and carbon. $\mathrm{CaCO}_{3}$-containing toothpastes have been shown to occlude the dentinal tubules and reduce dentin permeability. ${ }^{35}$ Furthermore, the average diameter of mid-coronal dentinal tubules ranged from 1.4 to $2.6 \mu \mathrm{m}$, so the prepared eggshell powder (where nanoclusters and nanoparticles with average size equal to $22.86 \mathrm{~nm}$ as revealed in our TEM micrographs) could have easily precipitated inside the orifices of dentinal tubules leading to their occlusion. Also, since nano-sized particles have a large surface area and high surface energy, they will have a high affinity to easily deposit on irregular spaces. ${ }^{31,36}$ This is in agreement with the findings of earlier studies that used nano-sized particles, e.g. nanohydroxyapatite, ${ }^{37}$ nano-carbonated apatite $^{36}$ and mesoporous silica nanoparticles ${ }^{20}$ for dentinal tubules occlusion. The significantly lower percentage of dentinal tubules occlusion after application of Tooth Mousse may be attributed to the assumption that a single application of Tooth Mousse was not as effective as a single application of the prepared eggshell slurry. It might have been unlikely to form stable calcium precipitates inside dentinal tubules following a single application of Tooth Mousse. ${ }^{38}$

After the acid challenge, the eggshell slurry significantly occluded more dentinal tubules compared to the Tooth Mousse. The difference in dentinal tubules occlusion might reflect differences in acid resistance of the precipitates formed by either desensitizing agent.

After immersion in artificial saliva, the eggshell subgroup showed a significantly higher mean percentage of dentinal tubules occlusion compared to that of the Tooth Mousse subgroup. On the contrary, the results of the percentage reduction of dentin permeability showed that the Tooth Mousse subgroup had a significantly higher median value of the percentage reduction of dentin permeability compared to that of the eggshell subgroup. This may be attributed to the deposition of newly formed precipitates on the specimens. ACP has the highest rate of formation and dissolution under physiologic oral conditions. It is also capable of conversion into hydroxyapatite crystals that can precipitate in the lumen of the opened dentinal tubules reducing their diameters. The CPP-ACP nanocomplexes will help in concentrating the bioavailable calcium and phosphate ions, and maintaining a state of supersaturation within the oral environment. 39,40

Also, after application of the agent and following the acid challenge, the results of the percentage reduction of dentin permeability revealed no significant difference between the prepared eggshell slurry and Tooth Mousse. However, the lower mean values of percentage of dentinal tubules occlusion after the acid challenge compared to that after application of both agents or after their immersion in artificial saliva as shown in the results and ESEM figures might denote that the formed mineral precipitates were not strong enough to withstand the acid challenge.
The discrepancy between the results of the percentage of dentinal tubules occlusion and the percentage reduction of dentin permeability may be considered as a limitation in our study. This was further confirmed by the insignificant negative correlation between the two tested techniques. Evaluation of the occluded dentinal tubules was carried out using ESEM where only the top dentinal surface was studied. The dentinal tubules in the eggshell slurry dentin disc specimens might have been occluded but not necessarily sealed, i.e., the eggshell slurry might have covered the dentin surface without completely sealing the dentinal tubules. However, Tooth Mousse might have precipitated deep in the tubules leaving the appearance of open dentinal tubules. This was in agreement with the findings of Zhang et al., which revealed that there did not seem to be a very good correlation between the SEM appearance of the dentin surfaces and its permeability. Surfaces covered by a thick amorphous material may have hydraulic conductance levels near the baseline value, indicating that the layer of the applied desensitizing agent is not necessarily sealing the tubules. ${ }^{41}$

Accordingly, we recommend that the hydraulic conductance test should be done conjointly with SEM analyses, since dentinal tubules may appear occluded, but not necessarily sealed.

Furthermore, the eggshell powder; such a calcium-rich natural biomaterial waste, could be used as a dentin desensitizing agent.

\section{Conclusion}

The avian eggshell slurry can effectively occlude open dentinal tubules compared to commercially available Tooth Mousse desensitizing agent. The avian eggshell slurry can be considered a promising material for the treatment of dentin hypersensitivity. Future in-vitro studies are required to evaluate the long-term efficacy of the eggshell slurry as a desensitizing agent after mechanical challenge (tooth brushing). Also, clinical studies analyzing the effect of eggshell slurry in treating dentin hypersensitivity are required.

\section{ORCID}

Aya A El Tahlawy @ 1 https://orcid.org/0000-0001-5131-9503

Dalia A Saba @ 1 https://orcid.org/0000-0001-7719-2635

Nahed G Bakir (1) https://orcid.org/0000-0001-8818-7540

\section{References}

1. Hongal S, Torwane NA, Goel P, et al. The effect of $30 \%$ ethanolic extract of Indian propolis on replica of human dentin compared against commercially available desensitizing agent: a methodological SEM study in vitro. Pharmacognosy Res 2014;6(2):113-119. Available from: http://www.phcogres.com/text.asp?2014/6/2/113/129026. DOI: 10.4103/0974-8490.129026.

2. Santiago SL, Pereira JC, Martineli AC. Effect of commercially available and experimental potassium oxalate-based dentin desensitizing agents in dentin permeability: influence of time and filtration system. Braz Dent J 2006;17(4):300-305. DOI: 10.1590/S010364402006000400007.

3. Dundar A, Yavuz T, Orucoglu H, et al. Evaluation of the permeability of five desensitizing agents using computerized fluid filtration. Niger J Clin Pract 2015;18(5):601-606. DOI: 10.4103/1119-3077.158949.

4. Torwane NA, Hongal S, Goel P, et al. A clinical efficacy of $30 \%$ ethenolic extract of Indian propolis and Recaldent ${ }^{\mathrm{TM}}$ in management of dentinal hypersensitivity : a comparative randomized clinical trial. Eur J Dent 2013;7(4):461-468. DOI: 10.4103/1305-7456.120675. 
5. Sasikumar S, Vijayaraghavan R. Low temperature synthesis of nanocrystalline hydroxyapatite from egg shells by combustion method. Trends Biomater Artif Organs 2006;19(2):70-73.

6. Durmuş E, Çelik I, Aydin MF, et al. Evaluation of the biocompatibility and osteoproductive activity of ostrich eggshell powder in experimentally induced calvarial defects in rabbits. J Biomed Mater Res B Appl Biomater 2008;86(1):82-89. DOI: 10.1002/jbm.b.30990.

7. Salama R, Khashaba M, El Rouby D. Histomorphometric evaluation of a nano-sized eggshell-containing supplement as a natural alloplast : an animal study. Saudi Dent J 2019;31(3):375-381. DOI: 10.1016/j. sdentj.2019.03.011.

8. Mony B, Rajesh Ebenezar AV, Ghani MF, et al. Effect of chicken egg shell powder solution on early enamel carious lesions: an invitro preliminary study. J Clin Diagn Res 2015;9(3):ZC30-ZC32. DOI: 10.7860/JCDR/2015/11404.5656.

9. Elsalamony NA, Abdel-hamid DM, Bakir NG. Bioactivity of a nanoeggshell-modified calcium hydroxide dental cement: an in-vitro study. J Dent Med Sci 2018;17(7):70-80. DOI: 10.9790/0853-1707037080

10. Allam G, El-geleel OA. Evaluating the mechanical properties, and calcium and fluoride release of glass-ionomer cement modified with chicken eggshell powder. Dent J 2018;6(3):40. DOI: 10.3390/dj6030040.

11. Onwubu SC, Vahed A, Singh S, et al. Reducing the surface roughness of dental acrylic resins by using an eggshell abrasive material. J Prosthet Dent 2017;117(2):310-314. Available from: http://linkinghub. elsevier.com/retrieve/pii/S0022391316303444. DOI: 10.1016/j. prosdent.2016.06.024.

12. Asghar W, Kim Y-T, llyas A, et al. Synthesis of nano-textured biocompatible scaffolds from chicken eggshells. Nanotechnology 2012;23(47):475601. Available from: http://www.ncbi.nlm.nih.gov/ pubmed/23111337. DOI: 10.1088/0957-4484/23/47/475601.

13. Salah M, Kataia MM, Kataia EM, et al. Evaluation of eggshell powder as an experimental direct pulp capping material. Futur Dent J 2018;4(2):160-164. DOI: 10.1016/j.fdj.2018.05.008.

14. Hassan TA, Rangari VK, Rana RK, et al. Sonochemical effect on size reduction of $\mathrm{CaCO}_{3}$ nanoparticles derived from waste eggshells. Ultrason Sonochem 2013;20(5):1308-1315. DOI: 10.1016/j. ultsonch.2013.01.016.

15. Mosaddegh E, Hassankhani A, Pourahmadi S, et al. Ball mill-assisted preparation of nano- $\mathrm{CaCO}_{3}$ as a novel and green catalyst-based eggshell waste: a green approach in the synthesis of pyrano[4,3-b]pyrans. Int J Green Nanotechnol 2013;1:1-5. DOI: 10.1177/1943089213507160.

16. UrazA, Gultekin SE, Senguven B, etal. Histologic and histomorphometric assessment of eggshell-derived bone graft substitutes on bone healing in rats. J Clin Exp Dent 2013;5(1):e23-e29. DOI: 10.4317/jced.50968.

17. Bashir ASM, Manusamy Y. Characterization of raw egg shell powder (ESP) as a good bio-filler. J Eng Res Technol 2015;2(1):56-60.

18. Thanatvarakorn O, Nakashima S, Sadr A, et al. In vitro evaluation of dentinal hydraulic conductance and tubule sealing by a novel calcium-phosphate desensitizer. J Biomed Mater Res B Appl Biomater 2013;101(2):303-309. DOI: 10.1002/jbm.b.32840.

19. Chen CLC, Parolia A, Pau A, et al. Comparative evaluation of the effectiveness of desensitizing agents in dentine tubule occlusion using scanning electron microscopy. Aust Dent J 2015;60(1):65-72. DOI: 10.1111/adj.12275.

20. Tian L, Peng C, Shi Y, et al. Effect of mesoporous silica nanoparticles on dentinal tubule occlusion: an in vitro study using SEM and image analysis. Dent Mater J 2014;33(1):125-132. Available from: http://jlc. jst.go.jp/DN/JST.JSTAGE/dmj/2013-215?lang=en\&from=CrossRef\& type=abstract. DOI: $10.4012 / \mathrm{dmj} .2013-215$.

21. Lata S, Varghese NO, Varughese JM. Remineralization potential of fluoride and amorphous calcium phosphate-casein phospho peptide on enamel lesions: an in vitro comparative evaluation. J Conserv Dent 2010;13(1):42-46. Available from: http://www.ncbi.nlm.nih.gov/ pubmed/20582219. DOI: 10.4103/0972-0707.62634.

22. Shi $S, W u Q, X u Y T$, et al. Long-term in vitro effectiveness of a bioglass desensitizing agent investigated using electrochemical impedance spectroscopy, atomic force microscopy and scanning electron microscopy. Dentistry 2017;7:4. DOI: 10.4172/2161-1122.1000422.
23. Pathan $A B$, Bolla $N$, Kavuri $S R$, et al. Ability of three desensitizing agents in dentinal tubule obliteration and durability: an in vitro study. J Conserv Dent 2016;19(1):31-36. DOI: 10.4103/0972-0707.173190.

24. Pashley DH, Galloway SE. The effects of oxalate treatment on the smear layer of ground surfaces of human dentine. Archs oral Biol 1985;30(10):731-737. DOI: 10.1016/0003-9969(85)90185-2.

25. Pinto SCS, Bandeca MC, Pinheiro MC, et al. Preventive effect of a high fluoride toothpaste and arginine-carbonate toothpaste on dentinal tubules exposure followed by acid challenge: a dentine permeability evaluation. BMC Res Notes 2014;7:385. DOI: 10.1186/1756-0500-7-385.

26. Thanatvarakorn O, Nakashima S, Sadr A, et al. Effect of a calciumphosphate based desensitizer on dentin surface characteristics. Dent Mater J 2013;32(4):615-621. Available from: http://www.ncbi.nlm.nih. gov/pubmed/23903644. DOI: 10.4012/dmj.2013-073.

27. Pereira JC, Segala AD, Gillam DG. Effect of desensitizing agents on the hydraulic conductance of human dentin subjected to different surface pre-treatments_an in vitro study. Dent Mater 2005;21(2):129138. DOI: 10.1016/j.dental.2004.02.007.

28. Sales-peres SHDC, Carvalho FN de, Marsicano JA, et al. Effect of propolis gel on the in vitro reduction of dentin permeability. J Appl Oral Sci 2011;19(4):318-323. DOI: 10.1590/s1678-77572011005000004.

29. Davari AR, Ataei E AH. Dentin hypersensitivity: etiology, diagnosis and treatment; a literature review. J Dent Shiraz Univ Med Sci 2013;14(3):136-145. PMCID: PMC3927677 PMID: 24724135

30. Kunam D, Manimaran S, Sampath V, et al. Evaluation of dentinal tubule occlusion and depth of penetration of nano-hydroxyapatite derived from chicken eggshell powder with and without addition of sodium fluoride: an in vitro study. J Conserv Dent 2016;19(3):239-244. Available from: http://www.jcd.org.in/text.asp?2016/19/3/239/181940. DOI: 10.4103/0972-0707.181940.

31. Khoozani NE, Bahrololoom ME, Bagheri R. Modification of a soft drink by adding calcium carbonate nanoparticles to prevent tooth erosion. J Dent Biomater 2014;1(2):38-44. Available from: https://doaj.org/ article/f18e41d19ea649b08ea06baeaba3726f

32. Onwubu SC, Mdluli PS, Singh S, et al. A novel application of nano eggshell/titanium dioxide composite on occluding dentine tubules: an in vitro study. Braz Oral Res 2019;33:1-10. DOI: 10.1590/18073107bor-2019.vol33.0016.

33. Machado AC, Rabelo FEM, Silva VM, et al. Effect of in-office desensitizers containing calcium and phosphate on dentin permeability and tubule occlusion. J Dent 2019;(May):1-7. DOI: 10.1016/j.jdent.2019.05.025

34. Freire MN, Holanda JNF, Administrativo C. Characterization of avian eggshell waste aiming its use in a ceramic wall tile paste. Cerâmica 2006;52(324):240-244. DOI: 10.1590/S0366-69132006000400004.

35. Wang Z, Sa Y, Sauro S, et al. Effect of desensitising toothpastes on dentinal tubule occlusion: a dentine permeability measurement and SEM in vitro study. J Dent 2010;38(5):400-410. DOI: 10.1016/j. jdent.2010.01.007.

36. Lee SY, Kwon HK, Kim BI. Effect of dentinal tubule occlusion by dentifrice containing nano-carbonate apatite. J Oral Rehabil 2008;35(11):847-853. DOI: 10.1111/j.1365-2842.2008.01876.x.

37. Yu J, Yang H, Li K, et al. A novel application of nanohydroxyapatite/ mesoporous silica biocomposite on treating dentin hypersensitivity: an in vitro study. J Dent 2016;50:21-29. DOI: 10.1016/j.jdent.2016.04.005.

38. Walsh LJ. The effects of GC Tooth Mousse on cervical dentinal sensitivity : a controlled clinical trial. Int Dent SA 2012;12(1):4-12.

39. Kowalczyk A, Botuliński B, Jaworska M, et al. Evaluation of the product based on Recaldent technology in the treatment of dentin hypersensitivity. Adv Med Sci 2006;51 Suppl 1:40-42.

40. Leila P, Shirin Z, Mahboubeh M, et al. Comparison the effect of casein phosphopeptide amorphous calcium phosphate and fluoride varnish on dentin hypersensitivity reduction. Casp J Dent Res 2015;4(2):20-26. DOI: 10.22088/cjdr.4.2.20

41. Zhang Y, Agee K, Pashley DH, et al. The effects of Pain-Free Desensitizer on dentine permeability and tubule occlusion over time, in vitro. J Clin Periodontol 1998;25(11 Pt 1):884-891. DOI: 10.1111/j.1600-051x.1998. tb02386.x. 